ONDERZOEKSRAPPORT NR 9301

From Decision Tables to Expert System Shells

by

J. VANTHIENEN

G. WETS

$D / 1993 / 2376 / 01$ 


\title{
From Decision Tables to Expert System Shells
}

\author{
J. VANTHIENEN \& G. WETS \\ Katholieke Universiteit Leuven \\ Department of Applied Economic Sciences \\ Dekenstraat 2, B-3000 Leuven (Belgium)
}

Phone: (0)16-28.58.09, Fax: (0)16-28.57.99, E-mail: fdban06@blekul11

\begin{abstract}
Maintaining knowledge based systems is not a trivial task. Because of the ability of the decision table technique to detect incompleteness and inconsistency, it has been recommended as a tool in verification and validation of knowledge bases.

In this paper, however, it is shown how decision tables can also be used to generate, and not just to validate, knowledge bases and how the transformation process from decision tables to knowledge base can be organized. Several options to generate rules or other knowledge representations from decision tables are described and evaluated. Various facilities are also considered to deal with consultation interfaces, explanation facilities, what-if simulations.
\end{abstract}

The proposed generation strategy enables the knowledge engineer to isolate the knowledge body from its implementation and from the specific consultation environment, and allows to concentrate on the acquisition and modelling issues. Because the application is generated from the decision tables, knowledge maintenance largely means table maintenance and the application can easily be transported to other tools or platforms. The generation process has been implemented for two commercial tools, AionDS and KBMS and has been applied to real world applications.

\section{Keywords}

Expert systems, decision tables, decision trees, knowledge acquisition, verification \& validation, rule minimization 


\section{Decision tables and Knowledge Based Systems}

A decision table is a tabular representation used to describe and analyze procedural decision situations, where the state of a number of conditions determines the execution of a set of actions. Not just any representation, however, but one in which all distinct situations are shown as columns in a table, such that every possible case is included in one and only one column (completeness and exclusivity).

"A decision table is a table, representing the exhaustive set of mutual exclusive conditional expressions, within a predefined problem area." [26]

The tabular representation of the decision situation is characterized by the separation between conditions and actions, on one hand, and between subjects and conditional expressions (states), on the other hand. Every table column (decision column) indicates which actions should (or should not) be executed for a specific combination of condition states.

In this definition, the decision table concept is deliberately restricted to the so called singlehit table, where columns are mutually exclusive. Only this type of table allows easy checking for consistency and completeness.

Decision tables and knowledge based systems show some striking similarities, although both approaches put strongly different emphases. While decision tables traditionally stress the representation facilities (with the resulting additional checking capabilities for completeness, consistency and correctness), knowledge based systems are mainly dealing with knowledge formulation (modularity, flexibility) and inference (performance, user friendliness).

Moreover, a lot of expert systems built nowadays are propositional expert systems, which are equivalent to decision tables [1]. All present efforts, however, to link knowledge based systems to decision tables, focus on the transformation from expert systems to decision tables, for reasons of validation, verification or execution efficiency. In [1] e.g., expert systems are transformed into decision tables, because execution of decision tables is much 
faster than that of the original expert system. This execution efficiency can even be optimized further as indicated in the section on optimization.

In this paper it is argued that there is no need to restrict the use of decision tables to the transformation from expert system to decision tables, in order to obtain smooth validation or execution efficiency. In our point of view it is clearly superior to model the knowledge by means of decision tables from the start, to validate and to optimize the tables and then to implement the system, e.g. using an expert system shell. In other words, the path from decision tables to knowledge based systems is as relevant as the reverse direction.

\section{What do decision tables offer more than verification and validation?}

There are three domains were decision tables can be used efficiently and effectively: during the knowledge aquisition process, during the verification and validation process and as a fast way of executing the expert system.

Decision tables offer more than validation and verification. Instead of building or generating decision tables only during the validation and verification process, they will also show significant advantages in the knowledge acquisition phase itself, in which all the information has to be transformed into a coherent substance (see e.g. [13]). In the knowledge acquisition stage, a series of decision tables can be built. These tables constitute a hierarchy, as each condition or action in a decision table might be elaborated in a lower condition or action subtable respectively. In this paper it is assumed that the decision tables have been built (cf. infra) and will be converted to a full knowledge based application.

When the decision tables are transformed into an expert system shell this can be done using if-then-else statements or by using rules, as indicated in section 4. It is important to notice that after this transformation process, there will always remain a mapping between the decision tables and the expert system. Changes will be made to the decision table and immediately the expert system will be adapted. In this way maintaining the knowledge base becomes easier. The generation process has been implemented for two commercial tools, AionDS and KBMS (fig. 1). 


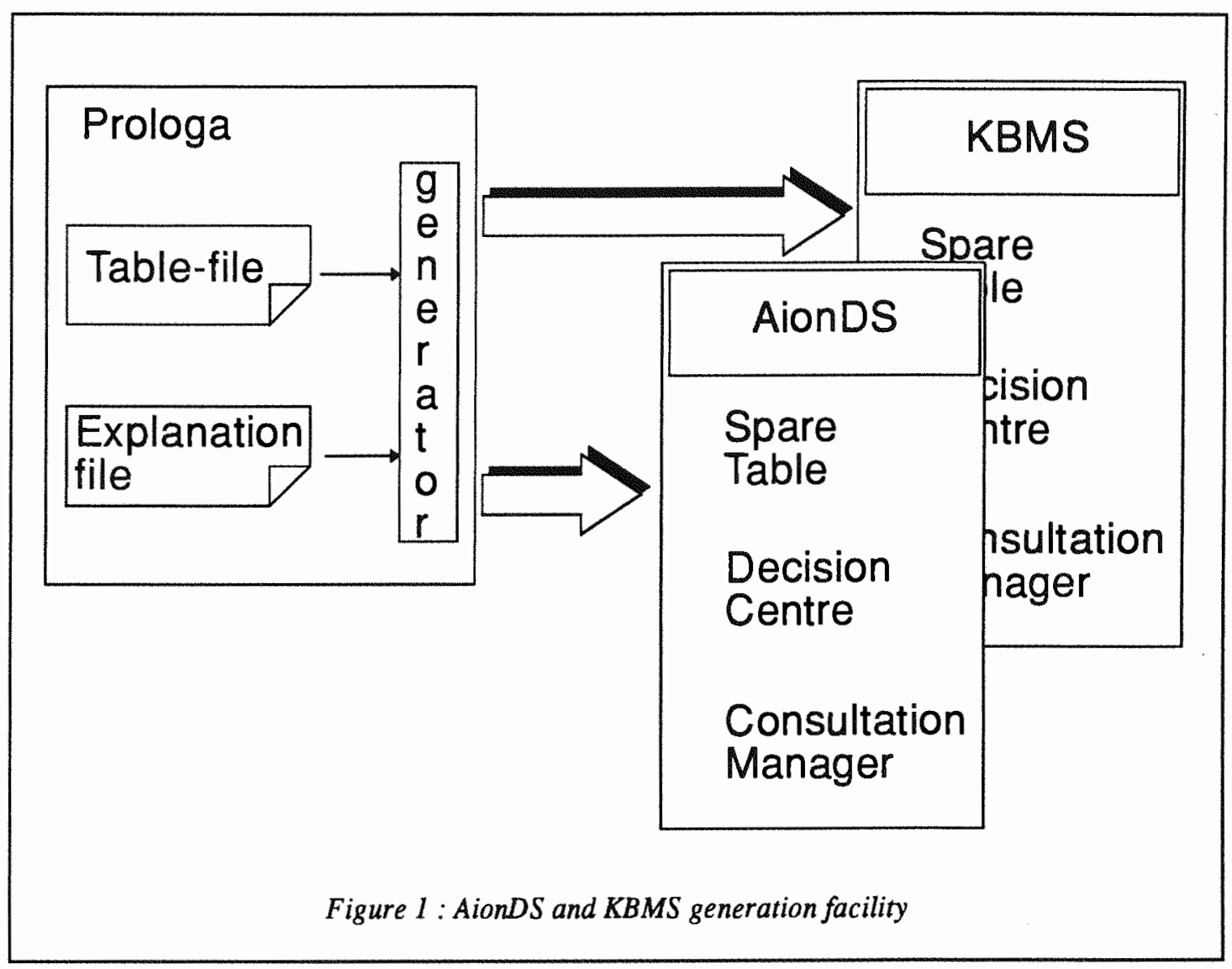

\section{Knowledge acquisition and verification \& validation}

\section{1. MODELLING WITH DECISION TABLES}

In this phase a hierarchy of decision tables will be built modelling the domain knowledge. The decision tables might for instance be built using the PROLOGa (PROcedural LOGic Analyzer) system, an interactive rule-based design tool for computer-supported construction and manipulation of decision tables ([23], [24]). This decision table engineering workbench incorporates powerful rule based knowledge acquisition and representation, table based verification, and adequate consultation interfaces to common shells and languages. How this hierarchy of decision tables is built will not be explained here because modelling is not the main issue of this paper (see e.g. [13] for further information about modelling with decision tables.) 


\section{2. VERIFICATION AND VALIDATION USING DECISION TABLES}

Gathering the knowledge is one of the main problems in building knowledge based systems, and usually, after the knowledge acquisition process is finished, a lot of contradictions and insufficiencies remain to be detected and solved. Also, maintaining the knowledge base is not a trivial task which often introduces unnoticed inconsistencies or contradictions.

A lot of current knowledge based systems, however, offer little or no guarantees to support validation, change and complexity control. Verification and validation of knowledge based systems are receiving increased attention [9], [4], [22], [18], [6], [15], [16], [7].

The emerging problems of validation and verification have led to the occasional use of schemes, tables or similar techniques in knowledge representation and validation. It has been reported earlier (e.g. [23], [3], [17], [1]) that, in a vast majority of cases, the decision table technique is able to provide for extensive validation and verification assistance. It easily enables the designer to check for contradictions, inconsistencies, incompleteness, redundancy, etc. in the problem specification. Most of the common validation problems [14] can easily be solved using decision tables (see e.g. [24]):

\section{- Consistency and Correctness of Knowledge}

Dividing knowledge over a large number of rules, designed independently, may lead to problems of inconsistency, such as: Conflict, Cyclical rules, Invalid attribute values, Unreachable conditions.

\section{- Non-redundancy of Knowledge}

Redundancy usually does not lead to errors during consultation of the system, but it may considerably harm efficiency. The main problem with redundancy, however, is not inefficiency, but maintenance and the risk of creating inconsistencies when changing the knowledge base. Current problems are: Subsumption, Redundant premises, Redundant rules. 


\section{- Completeness of Knowledge}

No current system is able to incorporate all possible knowledge, but within the specific problem area, the following omissions often occur: Missing knowledge, Unused attribute values or combinations, Unreachable conclusions.

One of the major advantages of the decision table approach is that checking for completeness and consistency can already be performed during the design of the knowledge based system. At the moment validation and verification of knowledge bases takes place after the construction process. It is a clearly better solution to detect errors as soon as possible. 


\section{Transformation framework}

This transformation framework consists of three points. First the different optimizations which can be applied will be described. Then the transformation of optimized decision tables to expert system shells will be explained. Finally the consultation environment is added.

\subsection{OPTIMIZATIONS USED IN THE TRANSFORMATION}

In the remainder of this section a simple decision table (figure 2) will be used as a starting point.

\begin{tabular}{|c|c|c|c|c|c|}
\hline 1. $\mathrm{C} 1$ & & Y & & & N \\
\hline 2. $\mathrm{C} 2$ & $Y$ & $\mathrm{~N}$ & & $Y$ & $\mathrm{~N}$ \\
\hline 3. c3 & \begin{tabular}{l|l}
$Y$ & $N$ \\
\end{tabular} & $\mathrm{Y} \mid \mathrm{N}$ & & $\mathrm{N}$ & $\mathrm{Y} \mid \mathrm{N}$ \\
\hline $\begin{array}{l}\text { 1. } \mathrm{A} 1 \\
2: \mathrm{A} 2 \\
3: \mathrm{A} 3\end{array}$ & $\left|\begin{array}{l}x \\
\dot{x}\end{array}\right| \dot{x}$ & $\begin{array}{l}x \\
:\end{array}$ & & $\dot{x}$ & $\dot{x} \mid \dot{x}$. \\
\hline
\end{tabular}

Figure 2: Expanded table

Note: The decision table concept in this paper is by no means restricted to limited-entry tables. Only, this example happens to consist of $\mathrm{Y} / \mathrm{N}$ entries.

The following optimizations can be performed starting from an expanded decision table:

- Table contraction: minimizing the number of columns for a given condition order by combining columns which lead to the same action configuration (figure 3) [23];

\begin{tabular}{|c|c|c|c|c|c|c|}
\hline 1. C1 & \multicolumn{4}{|c|}{$Y$} & & N \\
\hline 2. $\mathrm{C} 2$ & & $Y$ & & $\mathrm{~N}$ & & - \\
\hline 3. c3 & $Y$ & $\mathrm{~N}$ & $Y$ & $\mathrm{~N}$ & $Y$ & $\mathrm{~N}$ \\
\hline $\begin{array}{ll}1 \cdot A 1 \\
2 \cdot A & \text { A2 } \\
3 & \text { A3 }\end{array}$ & $\begin{array}{l}\mathrm{x} \\
\mathrm{x}\end{array}$ & $\dot{x}$ & $\begin{array}{c}\mathrm{X} \\
\text {. }\end{array}$ & $\dot{x}$ & & $\dot{x}$ \\
\hline
\end{tabular}

Figure 3: Table contraction 
- Row order optimization: determination of the condition order which results in the minimum number of columns. For a table with $\mathrm{N}$ conditions, this implies a choice between $\mathrm{N}$ ! alternative condition orders, some of which might be impossible because of precedence constraints. E.g., the table in figure 3 with 6 columns is optimized in figure 4. Now it only shows 4 columns ;

\begin{tabular}{|c|c|c|c|c|}
\hline 1. C3 & & $Y$ & & $\mathrm{~N}$ \\
\hline 2. $\mathrm{C} 1$ & & ? & $\mathrm{N}$ & - \\
\hline 3. C2 & $Y$ & $\mathrm{~N}$ & - & - \\
\hline $\begin{array}{ll}1 . & \text { A1 } \\
2 & \text { A2 } \\
3 & \text { A3 }\end{array}$ & $\begin{array}{l}x \\
x\end{array}$ & $\begin{array}{l}x \\
\text {. }\end{array}$ & $x$ & $\dot{x}$ \\
\hline
\end{tabular}

Figure 4: Row order optimization

Table contraction and row order optimization are important to enhance the effectiveness of the decision table in the user interface or to facilitate decision making [21], [27].

- Execution time optimization: depending on the purpose of the decision table, it might be transformed into optimal test sequences, taking into account condition test times and column frequencies (if available). In the optimal solution it is possible that conditions are not always tested in the same order. As a consequence it will, in general not be possible to present the optimal solution as a decision table, therefore the optimal solution will be presented as an unbalanced tree. In this case conditions are not always examined in the same order.

For a table with $n$ limited entry conditions, this implies a choice between $\mathrm{f}(n)$ decision trees, where:

$$
\begin{aligned}
\mathrm{f}(n) & =n \cdot[\mathrm{f}(n-1)]^{2}, \text { with } \mathrm{f}(1)=1 \\
& =n \cdot(n-1)^{2} \cdot(n-2)^{4} \cdot(n-3)^{8} \cdot .2^{2^{* *}(n-2)} \\
& =\prod_{\mathrm{i}=0}^{\mathrm{n}-1}(n-\mathrm{i})^{2^{\mathrm{i}}} \\
& =\prod_{\mathrm{j}=1}^{\mathrm{n}} \mathrm{j}^{2(n-\mathrm{j})}
\end{aligned}
$$


In decision table research, a lot of effort has been spent on generating this optimal tree (see e.g. [25], [11], [8], [19]). For the decision table of figure 2 (assuming equal column frequencies and equal condition test times) the optimal execution tree is displayed in figure 5. In this (exceptional) case, the tree is a balanced tree.

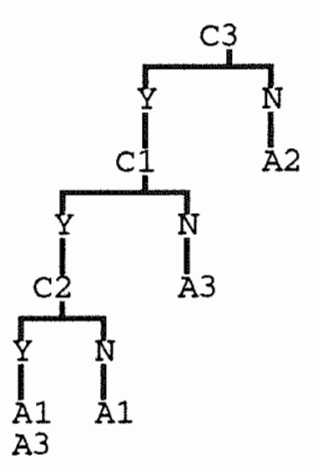

Figure 5: Optimal execution tree

Note that generating the optimal tree may have some major drawbacks and difficulties

1. How to determine testing costs of conditions?

2. How to determine column frequencies?

3. The performance of the conversion algorithm.

If testing costs and column frequencies are not available they are supposed to be equal. In this case execution time optimization minimizes the average number questions needed for making a decision.

- Minimization of expressions governing the same action:

A lot of research has already been performed in this area especially in the field of switching circuits. In this section a short overview of this research will be given.

In the 1950's, the starting years of digital design, logical gates were expensive. It was therefore necessary to produce, for a certain logic circuit, an equivalent circuit but with less electronic components. At that time simplifications of logic functions became a hot topic. First Karnaugh developed a pictorial method to obtain minimum logic functions. A similar method was developed by Veitch. These methods where convenient for 
problems up to 6 variables. Later on, more sophisticated methods where developed by Quine and McCluskey: the Quine-McCluskey method and the consensus method [12]. These methods involve two major steps :

First all prime implicants are generated. Let $\mathrm{A}$ be a logical expression. A fundamental conjunction $\psi$ is said to be a prime implicant of $\mathrm{A}$ if $\psi$ logically implies A while any other fundamental conjunction obtained by eliminating literals from $\psi$ does not logically imply A. A statement $X$ logically implies a statement $Y$ if and only if every assignment of truth values making $X$ true also makes $Y$ true.

The second step extracts a minimum prime cover. This is defined as a sum of product terms which has a minimum number of terms and of all those which have the same number of terms has a minimum number of assertions.

These methods were only practical for small problems (max. 10 variables). To solve more realistic problems, heuristics were developed. Two approaches can be distinguished here. One approach generates all prime applicants, like the QuineMcCluskey method, but instead of generating a minimum cover a nearly minimum cover is generated heuristically. A second approach tries to simultaneously identify and select implicants for the cover.

While originally research about this subject was conducted in the field of microelectronics, in the '70s also the A.I.-community started to show some interest in this problem. In decision tables reduction of rules governing the same action was investigated when minimizing a decision grid chart. The decision grid chart is a special kind of multiple hit table, in which the rules are grouped by action.

Strunz [20] applied the Quine-McCluskey algorithm to obtain a minimum decision grid chart to facilitate conversion into a contracted decision table. Applying this means that first a full expansion of the decision grid chart is necessary (containing no dashes as entries). To avoid this problem Maes [10] used the consensus method instead of the Quine-McCluskey method to find the prime implicants.

In our example, optimization of expressions governing the same action yields the following: 


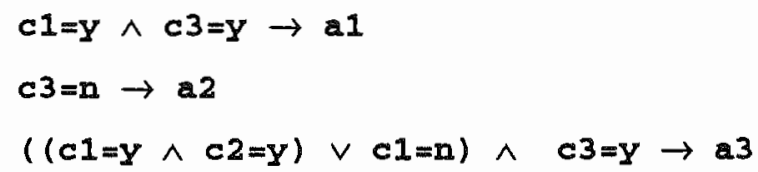

This kind of optimization can be useful when we want to reconstruct a specification based on actions (e.g. rewriting legal texts).

\subsection{FROM DECISION TABLES TO EXPERT SYSTEM SHELLS}

When designing or generating an application for an existing knowledge based tool, an object structure has to be developed: conditions, condition states, conclusions and table references have to be transformed to the available modelling facilities. This transition will not be considered here, as it is rather specific to the target environment. See section 5 for a transition to AionDS.

Once the object structure is built, the main problem is how to implement the decision logic knowledge which uses this object structure. The knowledge has been modelled in the form of decision tables (with proper verification and validation) and the table logic will be converted to the knowledge base. The implementation of the decision logic can be realized in two different ways. It is possible to convert the decision tables to a decision tree or to code using a nested if-then-else structure. The second way of implementing the decision tables is to convert them to a set of rules. Of course a combination of both can be useful sometimes.

\section{A. Conversion of decision tables to a decision tree or to code}

When the reasoning process is constant, the table might be transformed to a nested if-thenelse structure, where the outcome of the decision is obtained by choosing the appropriate branch in the selection. The decision logic can also be represented as a tree structure for pictorial reasons.

Within this option, several types of trees might be the result of the conversion: balanced trees (where conditions in the tree are always examined in the same order, if relevant) and unbalanced trees (where the order in which conditions are evaluated might differ between different branches). It will be clear that unbalanced trees offer more flexibility, but 
constitute a more complex optimization challenge. The different kinds of optimizations are treated more formally in section 4.1 .

\section{A naive balanced tree}

In this case the decision table is simply transformed into a tree from left to right, each column giving rise to a new leaf of the tree. The decision table in figure 3 will then be transformed to the following balanced tree (figure 6):

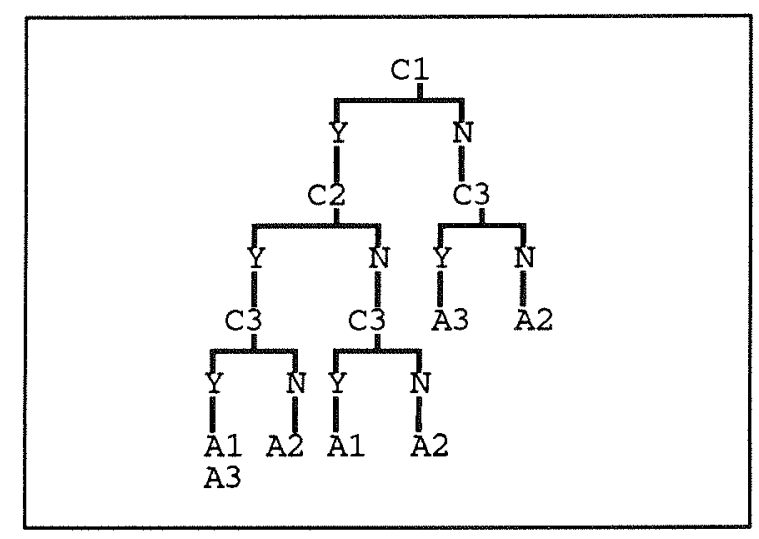

Figure 6: Decision tree of the contracted table

The corresponding if-then-else structure is:

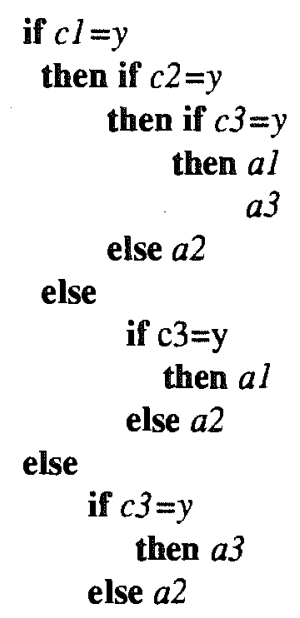

Figure 7: If-then-else structure of the contracted table 
A Minimal Node (balanced) tree is similar but has been optimized with respect to the number of leafs. This means that the minimal node tree is the tree resulting from the table with the minimum number of columns, selected from all feasible condition orderings. In this tree, conditions are still always examined in the same order, but the order might be different from that of the original decision table. This structure corresponds with the decision table in figure 4 and is displayed in figure 8 :

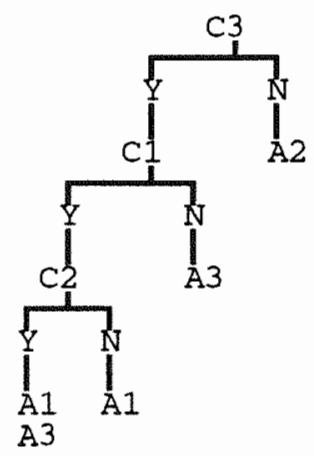

Figure 8: Minimal node tree

The if-then-else structure of this tree is :

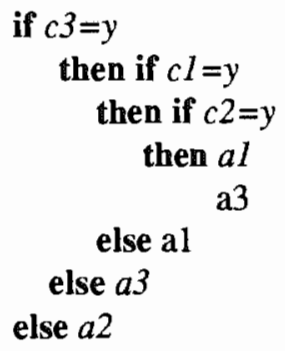

Figure 9: Minimal node conversion

\section{Optimal unbalanced tree}

This is the result of the execution time optimization from 4.1. In this exceptional case the optimal unbalanced tree happens to be the same as the minimal node balanced tree (figure 8) and therefore this figure will not be repeated. 


\section{B. Conversion of decision tables to a set of rules}

In a lot of cases, conversion to one decision statement is not flexible enough to deal with the knowledge in the knowledge base. One might prefer to transform the decision table into a set of rules. Several altematives are available:

1. Entry (x) based translation generates one rule for each $x$ in the (contracted) table. This method leads to several rules with the same premises, but different conclusions, if more then one action has to be performed for a certain combination of attributes. This of course results in a decrease in performance. The decision table in figure 3 will then be transformed to the following 7 rules (figure 10):

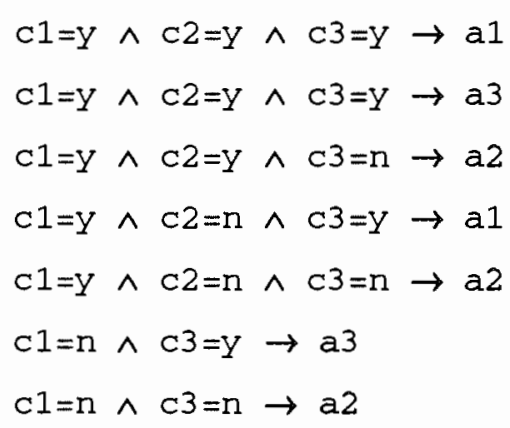

Figure 10: Entry based translation

2. Column (case) based translation generates a rule for each column of the decision table. All actions resulting from a specific combination of conditions will be included in one rule. The rules may even be combined further. This is interesting because every possible situation is represented by one rule.

Each of the three previous decision tree conversions can be used as a basis for rule generation. As each leaf in the decision tree will be represented by a rule, minimizing the number of leaves will minimize the number of rules. Optimality of the execution tree, however, does not necessarily imply optimal performance of the rule based system, because this will also depend on the specific matching and execution mechanisms.

The following rules can be deduced from the decision table in figure 4 .

$$
\mathrm{c} 3=\mathrm{y} \wedge \mathrm{c} 2=\mathrm{y} \wedge \mathrm{c} 1=\mathrm{y} \rightarrow \mathrm{a} 1 \wedge \mathrm{a} 3
$$




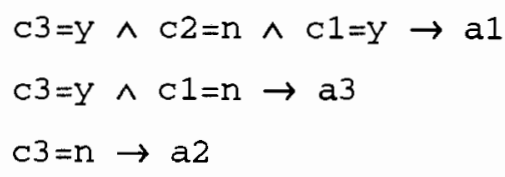

Figure 11: Column based translation

3. Row (action) based translation generates a rule for each relevant action (or nonempty row). The conversion from the decision table to the rules however is a little more complex, because rules for the same action have to be combined. When applying this method, the number of rules is usually very low, but the left hand side of the rules tends to be complex. Note that this specification corresponds well to the knowledge acquisition stage, as it describes the entire application field of a conclusion or action. In this case the optimization of the rules goveming the same action can be readily deduced. In more difficult examples however optimization algorithms are used to obtain the optimal rule set, see section 4 .

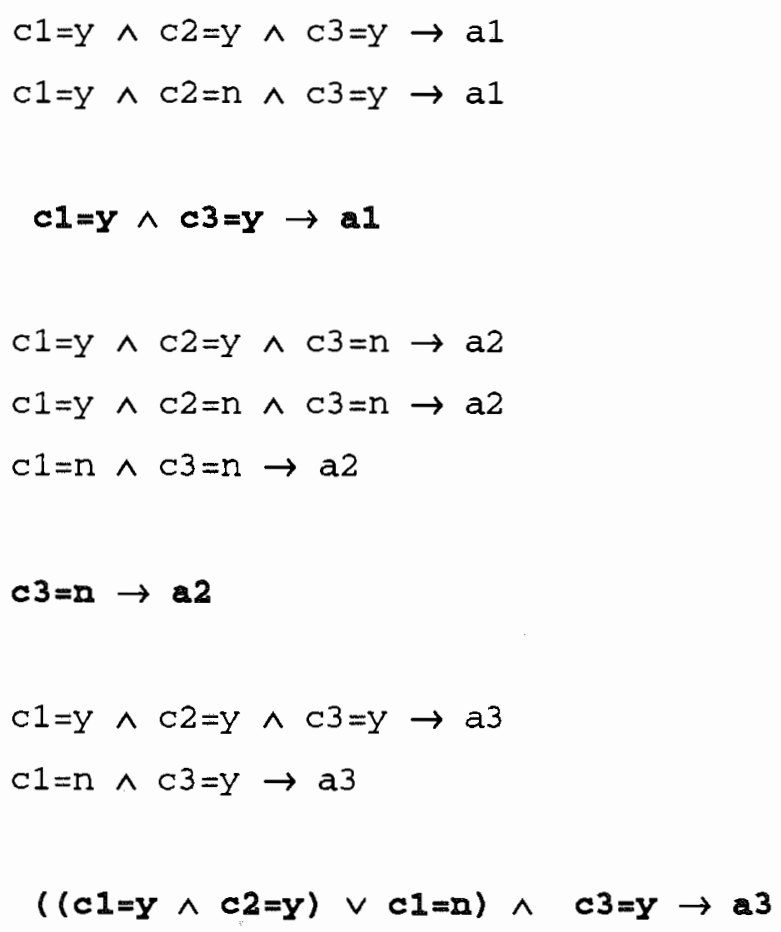

Figure 12: Row based translation 


\subsection{CONSUltation ENVIRONMENT}

The consultation environment offers the necessary user and system interfaces to produce a working application. Its implementation will depend on the tool used, but is not problemspecific.

The hierarchy of decision tables is translated into a question and answer interface. The user, however, need not be aware of the existence of the decision tables or any relations between them. In translating the hierarchy, attention must be paid that no circular inferencing arises, that recursive table calls remain possible and that condition subtable results are properly assigned to the calling conditions.

The following demands will have to be met by the consultation environment:

- The consultation environment has to be as independent as possible from the decision tables. Therefore the environment can be built in a generic way and according to specific user interface standards.

- The user interface must allow easy communication with the user, e.g. using multiple windows, mouse support, etc.

- Prompt and help texts should be available for conditions, condition states and actions, to explain the meaning of questions and conclusions.

- At any moment a list of questions and supplied answers can be shown with the ability to change previous answers and restart the reasoning process from the specified point (WHAT IF simulations).

- At any moment it must be possible to leave the application (saving the current contents of the consultation) and restart it later. This also enables to store a list of prototype cases which can be adapted using the selective change facility.

- Maintaining the knowledge base must be easy. It should for instance be possible to plug in an updated decision table without having to generate the complete application again.

\section{An Example: the Transformation Prologa-AionDS}

The above transformation process has been implemented in the decision table engineering workbench PRologa (PROcedural LoGic Analyzer, [24]). An interface was built between 
PROLOGA and AionDS (Aion Development System, AION), [5]. This enables the knowledge engineer to model and validate the knowledge in the form of decision tables and generate a complete consultation application in AionDS. For an interface between Prologa and KBMS (Knowledge Based Management System, AICORP), see [2].

\subsection{KNOWLEDGE STRUCTURE}

\section{A. Available source elements}

When transforming the decision tables, the following information is available:

- Names of conditions, condition states and actions. References to subtables are indicated in the condition or action names.

- The (contracted) decision tables on a column by column basis, where every column indicates a combination of conditions with the resulting action configuration.

- Every table can also be provided with an explanation file containing prompts, help texts or other information specific to the consultation. The explanation file is basically independent from the decision logic, such that the decision table can be altered without having to update the help information.

AionDS, being object oriented, uses the concept of classes consisting of a set of objects which have attributes called slots. An AionDS application is a hierarchy of states, where in each state rules, functions and an agenda can be defined.

\section{B. Generation of a decision centre}

Each decision table is converted to a class and condition and action variables are converted to slots, which may be linked to other conditions or actions. The decision table is converted to if-then-else code as a function in a class. All subtables in a hierarchy are generated and linked automatically. 


\subsection{IMPLEMENTATION OF THE KNOWLEDGE STRUCTURE}

The interface offers three related generation options: a decision centre, the consultation manager and the spare table option.

\section{Decision Centre}

This option only generates the basic knowledge structure: a class with the converted actions, condition variables and decision table logic. No additional consultation facilities are provided. This option can be used if you want to make use of consultation options offered by the expert system shell itself or use your own specific environment.

\section{The Consultation Manager}

A full consultation environment is built together with the decision table application. This includes the following facilities:

- View: a list of all variables with their values.

- Footsteps: a chronological survey of the questions asked, with the answer and the conclusions reached by the expert system.

- WhatIf-mode and Reconsider: offers the possibility in Footsteps or View to change one or more answers and restart the reasoning process.

\section{Spare Table}

This option enables the designer to plug in the decision logic of an updated table into an existing application, without having to repeat the entire generation process, and leaves the rest of the application intact.

\section{Conclusion}

In this paper it has been shown that decision tables offer more than verification and validation. A transformation framework was developed to incorporate the decision tables in expert system shells. This transformation framework was first explained theoretically. The different optimizations which can be applied, the different ways of implementing the 
knowledge structure and the consultation environment were described. Finally this transformation framework was implemented for the specific expert system shell AionDS.

\section{Acknowledgements}

The authors wish to thank M. Verhelst for his comments on an earlier version of this paper. They also greatly acknowledge the invaluable assistance of S. Jans, P. Merlevede, and G. Van Humbeeck. This research was conducted in the context of LIRIS (Leuven Institute for Research on Information Systems). 


\section{References}

[1] Colomb R. and Chung C., Very Fast Decision Table Execution of propositional Expert Systems (Proc. AAAI90 1990) 671-676.

[2] Coucke B., Ontwikkeling van een interface Prologa-KBMS (Knowledge Base Management System), K.U.Leuven TEW dissertation, 1992.

[3] Cragun B. and Steudel H., A Decision-Table Based processor for Checking Completeness and Consistency in Rule-Based Expert Systems, Int. Joumal of ManMachine Studies 5 (1987) 633-648.

[4]Hamilton D., Kelley K. and Culbert C., State-of-the-Practice in Knowledge-Based System Verification and Validation, Expert Systems with Applications 4 (1991) 403410.

[5] Jans S., Een interface tussen Prologa en AionDS, Toepassing van de beslissingstabellenbenadering bij het ontwerp en de implementatie van een kennissysteem, K.U.Leuven TEW dissertation, 1992.

[6] Lalo A., TIBRE: Un Système Expert Qui Teste Les Incoherences Dans les Bases de Règles in (Proc. Avignon88 Vol. 3 1988) 63-84.

[7] Larsen H. and Nonfjall H., Modeling in the Design of a KBS Validation System, Int. Journal of Intelligent Systems 6 (1991) 759-775.

[8] Lew A., Optimal Conversion of Extended-entry Decision Tables with General Cost Criteria, Comm. of the ACM 4 (1978) 269-279.

[9] Loveland D. and Valtorta M., Detecting Ambiguity : An Example in Knowledge Evaluation, in: Proc. Eighth Int. Joint Conf. on Artificial Intelligence, Karlsruhe, Aug. 1983, Vol. 1, 182-184.

[10] Maes R., On Minimizing Decision Grid Charts, Angewandte Informatik 9 (1982) 451456.

[11] Martelli A. and Montanari U., Optimizing Decision Trees through Heuristically Guided Search, Comm. of the ACM 12 (1978) 1025-1039.

[12] McCluskey E. J., Introduction to the theory of switching circuits, (Mc.Graw-Hill, New York, 1965). 
[13] Merlevede P. and Vanthienen J., A Structured Approach to Formalization and Validation of Knowledge, in Proc IEEE/ACM Intemational Conference on Developing and Managing Expert System Programs, Washington, DC, 1991, 149-158.

[14] Nguyen T., Perkins W., Laffey T. and Pecora D., Knowledge Base Verification, AI Magazine 2 (1987) 69-75.

[15] Nonfjall H. and Larsen H., Detection of Potential Inconsistencies in Knowledge Bases, Int. Journal of Intelligent Systems 7 (1992) 81-96.

[16] O'Leary T. and Goul M. et al., Validating Expert Systems, IEEE Expert 3 (1990) 51-58.

[17] Puuronen S., A Tabular Rule Checking Method, in: Proc. Avignon87, Vol. 1, 1987 257268.

[18] Rousset M., Sur La Validité Des Bases de Connaissances: le Système COVADIS, Proc. Avignon87, Vol. 1, 1987 269-282.

[19] Sethi I. and Chatterjee B., Conversion of Decision Tables to efficient Sequential Testing Procedures, Comm. of the ACM 5 (1980) 279-285.

[20]Strunz H., Grundlagen und Anwendingsmöglichkeiten der Entscheidungs tabellentechnik bei der Gestaltung rechnergestützter Informationsysteme, University of Köln, 1975.

[21] Subramanian G.H. and Nosek J. et al., A Comparison of the Decision Table and Tree, Comm. of the ACM 1 (1992) 89-94.

[22] Suwa M., Scott A. and Shortliffe E., Completeness and Consistency in a Rule-Based System, in : BUCHANAN \& SHORTLIFFE , 159-170.

[23] Vanthienen J., Automatiseringsaspecten van de specificatie, constructie en manipulatie van beslissingstabellen, K.U.Leuven Dept. Applied Econ. Doctoral Dissertation, 1986.

[24] Vanthienen J., Knowledge Acquisition and Validation Using a Decision Table Engineering Workbench, The World Congress on Expert Systems, Pergamon Press, Orlando (Florida), 16-19/12/91, 1861-1868.

[25] Verhelst M., The Conversion of Limited-Entry Decision Tables to Optimal and NearOptimal Flowcharts: Two New Algorithms, Comm. of the ACM 11 (1972) 974-980.

[26] Verhelst M., De Praktijk van Beslissingstabellen, Kluwer, Deventer/Antwerpen, 1980.

[27] Vessey, I. and Weber, R., Structured tools and conditional logic: An empirical investigation, Comm. of the ACM 1 (1986), 48-57. 


\section{Contents}

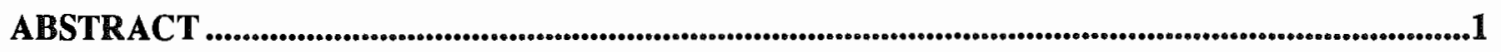

1. DECISION TABLES AND KNOWLEDGE BASED SYSTEMS....................................................2

2. WHAT DO DECISION TABLES OFFER MORE THAN VERIFICATION AND

VALIDATION? ...........................................................................................................................................................

3. KNOWLEDGE ACQUISITION AND VERIFICATION \& VALIDATION........................................4

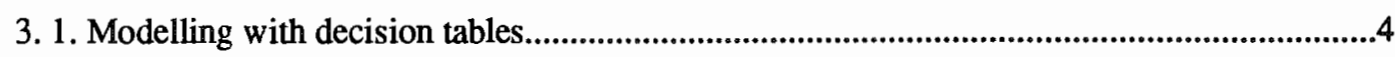

3. 2. Verification and Validation using decision tables.............................................................5

4. TRANSFORMATION FRAMEWORK ................................................................................

4.1. Optimizations used in the transformation ..........................................................................

4.2. From decision tables to expert system shells .......................................................................11

A. Conversion of decision tables to a decision tree or to code ...............................................11

B. Conversion of decision tables to a set of rules ................................................................14

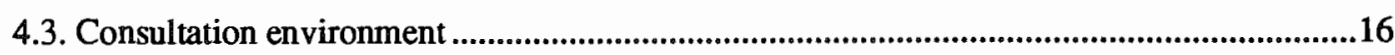

5. AN EXAMPLE: THE TRANSFORMATION PROLOGA-AIONDS.............................................16

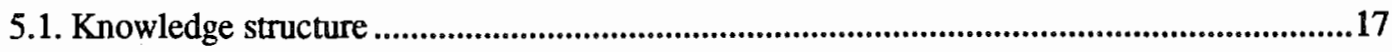

A. Available source elements ...............................................................................17

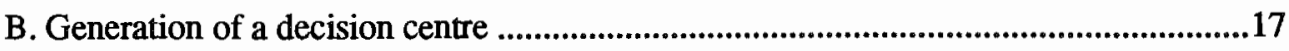

5.2. Implementation of the knowledge structure...................................................................18

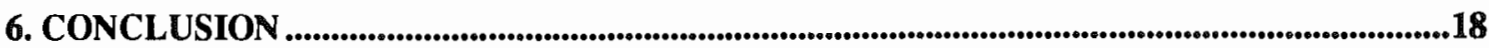

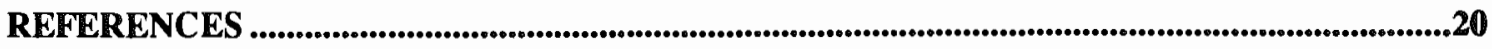

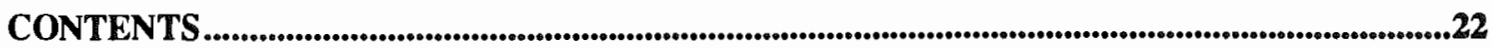

